TITLE:

\title{
Effect of the graft chain length and density on the morphology of radiation-modified polysilane monolayers at the air/water interface.
}

\section{$\operatorname{AUTHOR(S):~}$}

Tanaka, Hidenori; Kunai, Yuichiro; Sato, Nobuhiro; Matsuyama, Tomochika

\section{CITATION:}

Tanaka, Hidenori ... [et al]. Effect of the graft chain length and density on the morphology of radiation-modified polysilane monolayers at the air/water interface.. Journal of colloid and interface science 2011, 363(2): 440-445

\section{ISSUE DATE:}

2011-11-15

URL:

http://hdl.handle.net/2433/147227

\section{RIGHT:}

@ 2011 Elsevier Inc.; この論文は出版社版でありません。引用の際には 出版社版をご確認ご利用ください。; This is not the published version. Please cite only the published version. 


\section{Effect of the Graft Chain Length and Density on the Morphology of Radiation-Modified Polysilane}

\section{Monolayers at the Air/Water Interface}

Hidenori Tanaka, Yuichiro Kunai, Nobuhiro Sato*, and Tomochika Matsuyama

Research Reactor Institute, Kyoto University, Asashironishi 2-1010, Kumatori-cho, Sennan-gun, Osaka 590-0494, Japan

Corresponding author : Nobuhiro Sato

e-mail : sato-n@rri.kyoto-u.ac.jp

Phone: +81-72-451-2499

Fax: +81-72-451-2633

\section{Abstract}

The variation in the morphology of monolayers at the air/water interface is investigated for two kinds of radiation-modified polysilanes with different structures: poly(diethyl fumarate)-grafted poly(methyl- $n$-propylsilane) (PMPrS- $g$-PDEF) and maleic anhydride-grafted PMPrS (PMPrS- $g$-MAH). PMPrS- $g$-PDEF has long but sparsely-attached PDEF graft chains, while PMPrS-g-MAH has short but densely-attached MAH graft units. Surface pressure-area measurements indicate that PMPrS- $g$-PDEF monolayers extensively spread at the air/water interface though PMPrS homopolymer hardly spreads. AFM observation reveals that PMPrS-g-PDEF 
monolayers have an inhomogeneous structure containing string-like microstructures. This result suggests that PMPrS main chains are detached from the water surface to aggregate together and only PDEF chains spread over the water surface. In contrast, PMPrS- $g$-MAH forms uniform monolayers with a smooth surface. PMPrS main chains of PMPrS-g-MAH are anchored to the water surface by densely grafted MAH units. It is also demonstrated that only the PMPrS-g-MAH monolayers are successfully deposited layer-by-layer on a solid substrate by the Y-type deposition.

\section{Graphical abstract}

AFM observation reveals that PMPrS- $g$-PDEF monolayers have an inhomogeneous structure containing string-like microstructures. In contrast, PMPrS-g-MAH forms uniform monolayers with a smooth surface.

\section{Keywords}

Polysilanes / Graft Copolymers / $\gamma$-Rays / Radiation Modification / Monolayers / LB films

\section{Introduction}

Polysilane has received considerable attention in the past decades because it has various unique properties [1-12]. Among them, the optical properties are quite interesting because polysilane possesses a strong absorption band in the UV region due to $\sigma-\sigma^{*}$ transition of delocalized electrons along the silicon backbone $[1,13]$. This absorption band is concerned with the conformation of the backbone. For example, the absorption peaks of poly(methyl-n-propylsilane) (PMPrS) appearing at 305 and 325 
$\mathrm{nm}$ correspond to a random and a quasi-all-trans conformations, respectively. The latter conformation consists of an intermediate conformation between a helical form and the all-trans one, that is, a $\mathrm{D}+\mathrm{T}+\mathrm{D}-\mathrm{T}-$ repeated construction $(\mathrm{D}$, deviant; $\mathrm{T}$, transoid) [14].

Because of this unique property, the application of polysilane for ordered materials in the nanometer scale is an interesting theme both scientifically and technologically [15-17]. In our previous study, we found that radiation-modified polysilane forms nanoscale micelles in selective solvents $[18,19]$. As another method to construct nano-ordered structures of polysilane, the Langmuir-Blodgett (LB) film with amphiphilic polysilane is a promising candidate.

The LB film is an ultra thin film with well-defined layer structure fabricated through layer-by-layer deposition of monolayers at the air/water interface. Amphiphilic polysilanes to form monolayers at the air/water interface have been intensely studied [20-24], but there is no report about how the structure of amphiphilic polysilanes affects the morphology of their monolayers.

Until now, we have been studying the $\gamma$-ray-induced grafting of amphiphilic monomers onto hydrophobic polysilane to obtain amphiphilic polysilanes by a simple and easy technique under moderate chemical condition. In the course of this study, we found that amphiphilic polysilanes with different structures can be obtained by changing a type of grafted monomers [25]. While poly(diethyl fumarate)-grafted PMPrS (PMPrS-g-PDEF) has long but sparsely grafted chains, maleic anhydride-grafted PMPrS (PMPrS-g-MAH) has short (just one MAH unit) but densely grafted units. From this fact, it can be expected that the morphology of monolayers formed by these amphiphilic polysilanes is quite different according to the difference of graft chain 
length and density. In order to obtain high quality polysilane LB films, it is essential to establish optimum way of radiation-modification of polysilanes.

To clarify the effect of the grafting manner of amphiphilic polysilanes on the morphology of their monolayers at the air/water interface, surface pressure-area $(\pi-\mathrm{A})$ isotherms of the monolayers at the air/water interface and AFM images for the monolayers transferred onto solid substrates are closely examined in this study. Moreover, the deposition of LB films is demonstrated to construct the nano-ordered structure of polysilane.

\section{Experimental}

\subsection{Synthesis and characterization of PMPrS and radiation-modified PMPrS.}

The preparation and characterization of PMPrS- $g$-PDEF and PMPrS- $g$-MAH are as follows. Details of the synthetic procedure are described in our previous paper [25].

PMPrS was synthesized via the Wurtz-type coupling reaction with distilled methyl- $n$-propyldichlorosilane (Shin-Etsu Chemical, Co.). Number average molecular weight determined by GPC with an RI detector is shown in Table 1.

PMPrS- $g$-PDEF was synthesized through $\gamma$-ray-induced graft polymerization. PMPrS was dissolved together with diethyl fumarate (DEF; Nakalai Tesque, Inc.) in toluene and then irradiated with $\gamma$-rays after degassing (PMPrS $10 \mathrm{wt} \%$, DEF $30 \mathrm{wt} \%$, dose rate $2.0 \mathrm{kGy} / \mathrm{h}$ ). PMPrS-g-MAH was also prepared in a similar manner (PMPrS $10 \mathrm{wt} \%$, MAH $10 \mathrm{wt} \%$, dose rate $2.0 \mathrm{kGy} / \mathrm{h}$ ). Irradiation conditions and the number average molecular weights of the radiation-modified polysilanes are summarized in Table 1. Grafting yield, which is defined as the number of DEF or MAH units per silicon atom of PMPrS, was calculated from the integrated intensity ratio of ${ }^{1} \mathrm{H}$ NMR 
signals. Grafting yields of PMPrS- $g$-PDEF and PMPrS- $g$-MAH are also listed in Table 1.

The graft density of PMPrS- $g$-PDEF was evaluated from ${ }^{1} \mathrm{H}$ NMR spectra by quantitatively analyzing signals of the unsaturated end of PDEF graft chains. As a result, the number of PDEF graft chains per PMPrS main chain was found to be less than 1.0. On the other hand, PMPrS-g-MAH was more densely grafted. For example, PMPrS- $g$-MAH ${ }_{0.24}$ has one MAH unit per 4.2 silicone atoms, in other words, the number of MAH bonded to one PMPrS chain is $c a .30$ units.

Table 1. Number average molecular weight $\left(M_{\mathrm{n}}\right)$ and grafting yield of the radiation-modified polysilanes on various doses.

\begin{tabular}{|c|c|c|c|}
\hline & Dose (kGy) & $M_{\mathrm{n}}^{\mathrm{a}}$ & Grafting yield $^{b}$ \\
\hline PMPrS & & $1.2 \times 10^{4}$ & \\
\hline PMPrS- $g-\mathrm{PDEF}_{0.07}$ & 20 & $1.1 \times 10^{4}$ & 0.07 \\
\hline PMPrS- $g-\mathrm{PDEF}_{0.11}$ & 40 & $9.4 \times 10^{3}$ & 0.11 \\
\hline PMPrS- $g-$ PDEF $_{0.22}$ & 80 & $7.8 \times 10^{3}$ & 0.22 \\
\hline PMPrS- $g-$ PDEF $_{0.34}$ & 124 & $6.7 \times 10^{3}$ & 0.34 \\
\hline PMPrS & & $1.1 \times 10^{4}$ & \\
\hline PMPrS- $g-\mathrm{MAH}_{0.05}$ & 20 & $1.1 \times 10^{4}$ & 0.05 \\
\hline PMPrS- $g-\mathrm{MAH}_{0.08}$ & 40 & $1.0 \times 10^{4}$ & 0.08 \\
\hline PMPrS- $g-\mathrm{MAH}_{0.15}$ & 80 & $8.8 \times 10^{3}$ & 0.15 \\
\hline PMPrS- $g-M^{-} H_{0.24}$ & 140 & $6.6 \times 10^{3}$ & 0.24 \\
\hline
\end{tabular}


a Determined by GPC (RI detector) with polystyrene standards and a THF eluent.

Determined by ${ }^{1} \mathrm{H}$ NMR measurement.

\subsection{Film preparation.}

A spin-coated film was prepared on a quartz substrate from a toluene solution of PMPrS homopolymer or the radiation-modified PMPrS.

Monolayers of the radiation-modified PMPrS on solid substrates were prepared in the following manner: A benzene solution of $0.1 \mathrm{~g} / \mathrm{L}$ of the radiation-modified PMPrS was spread on the water surface. A monolayer at the air/water interface was compressed up to a given surface pressure in a similar manner described in the section of $\pi$-A isotherm measurements. Then, the monolayer was transferred onto a hydrophilic quartz substrate, which was facing perpendicular to the compression direction, by the up-stroke of the vertical dipping technique at a speed of $5 \mathrm{~mm} / \mathrm{min}$. Only for AFM observation, the monolayer was transferred onto a hydrophilic silicon substrate.

LB films were fabricated by the Y-type deposition of the monolayers at the air/water interface. Hydrophilic quartz substrates facing perpendicular to the compression direction were dipped at a speed of $5 \mathrm{~mm} / \mathrm{min}$ for both up- and down-strokes.

\section{Measurements.}

\section{1. $\pi$-A isotherm.}

A benzene solution of $0.1 \mathrm{~g} / \mathrm{L}$ of PMPrS or the radiation-modified PMPrS was spread on the water surface at $20^{\circ} \mathrm{C}$. The water for subphase was purified by deionizing with a Millipore filtration system after distillation. The monolayer at the air/water interface 
was compressed at a speed of $20 \mathrm{~cm}^{2} / \mathrm{min}$ and the $\pi$-A isotherm was monitored with a Wilhelmy plate.

\subsection{AFM observation.}

AFM observation was performed with a Shimadzu SPM 9500J system by the contact mode at room temperature. The spring constant of a micro-cantilever was $0.02 \mathrm{~N} / \mathrm{m}$. The transfer ratio of the monolayers used for AFM observation was 1.0 in all experiments.

\subsection{UV absorption spectroscopy.}

UV absorption spectra of spin-coated and LB films were recorded at room temperature with a Hitachi U-3400 spectrophotometer. In situ UV absorption spectra of monolayers at the air/water interface were obtained with the apparatus as shown in Figure 1. Light from a $\mathrm{D}_{2}$-lamp was guided over the water surface with an optical fiber. The light passing through a monolayer was reflected by a mirror at the bottom of a trough and again passed through the monolayer. Then, the light was captured and led to a detector with another optical fiber. As the light source and the detector, a Shimazu UV-3500PC spectrophotometer was used.

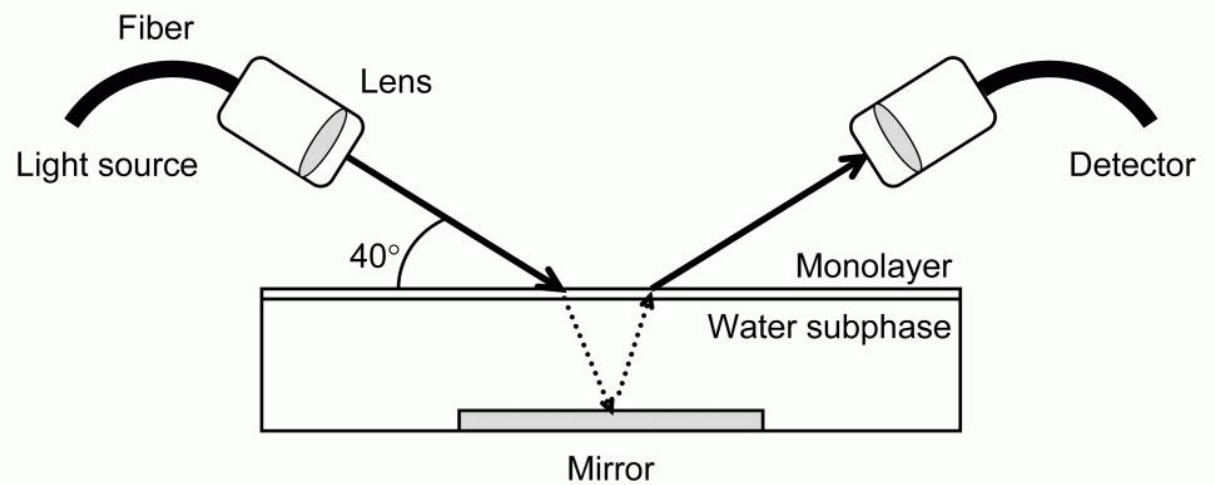


Figure 1. Apparatus for in-situ UV absorption spectroscopy of a monolayer at the air/water interface.

\section{Results and discussion}

\subsection{Conformation of PMPrS in PMPrS-g-PDEF spin-Coated Films}

The absorption intensity of PMPrS- $g-\mathrm{PDEF}_{0.34}$ spin-coated films around $300 \mathrm{~nm}$ was a little larger than that of PMPrS homopolymer, which means that PDEF graft chains slightly inhibit crystallization of PMPrS main chains to increase the fraction of the random conformation of PMPrS. However, it was also confirmed that UV absorption spectra of PMPrS- $g$-PDEF do not change in the range of grafting yield listed in Table 1. From these facts, it is supposed that PMPrS main chains and PDEF graft chains separately form their domains in the film.

\subsection{Monolayers and LB films of PMPrS-g-PDEF}

Figure 2 shows $\pi$-A isotherms of PMPrS- $g$-PDEF monolayers at the air/water interface. The isotherm of PMPrS homopolymer shows a very condensed profile because hydrophobic PMPrS cannot spread over the water surface and aggregates together. On the other hand, $\pi$-A isotherms of PMPrS- $g$-PDEF display more expanded profiles with an increase of grafting yield. The area at the lift-off point of the $\pi$-A isotherm has nearly linear relationship with the composition of DEF units. This result offers a piece of evidence that PDFE graft chains have the dominant effect on the behavior of PMPrS- $g$-PDEF monolayers at the air/water interface. 


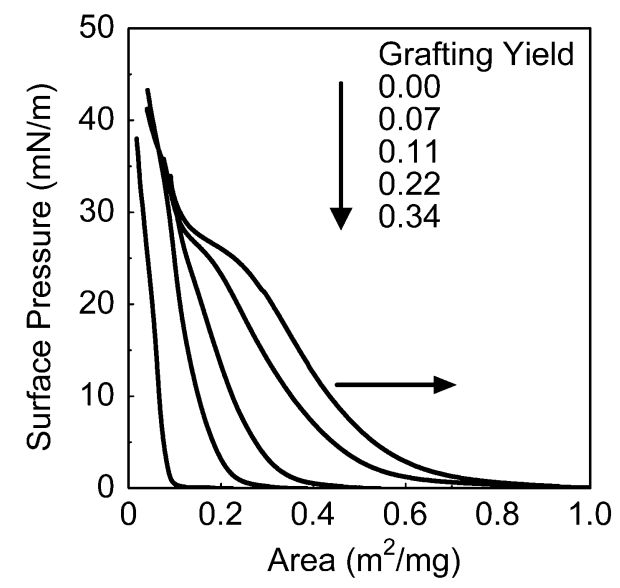

Figure 2. $\pi$-A isotherms of PMPrS- $g$-PDEF monolayers at the air/water interface.

The collapse pressure of PMPrS- $g$-PDEF monolayers also shows that PDEF chains have the most influence over their interfacial behavior. The collapse pressure of the monolayer of PDEF homopolymer was $28 \mathrm{mN} / \mathrm{m}$ in our another $\pi$-A measurement, which is consistent with those of PMPrS- $g-$ PDEF $_{0.22}$ and PMPrS- $g-P_{D E F}$ P.34 $_{1}$ monolayers as seen in Figure 2.

Next, AFM observation was carried out to reveal the morphology of PMPrS-g-PDEF monolayers transferred onto silicon substrates below the collapse pressure. AFM images of PMPrS- $g$-PDEF 0.22 monolayers are displayed in Figure 3. Both images indicate inhomogeneous features containing string-like microstructures. The density of the microstructures becomes higher with an increase of surface pressure. This morphology is formed due to the structure made of three-dimensionally contracted hydrophobic PMPrS chains escaping from the water surface and amphiphilic PDEF chains spreading over the water surface. This consideration is confirmed also by the fact that the height of the string-like microstructure evaluated from the AFM line profile 
(3-5 nm) is comparable with the root-mean-square diameter of PMPrS used in this experiment.
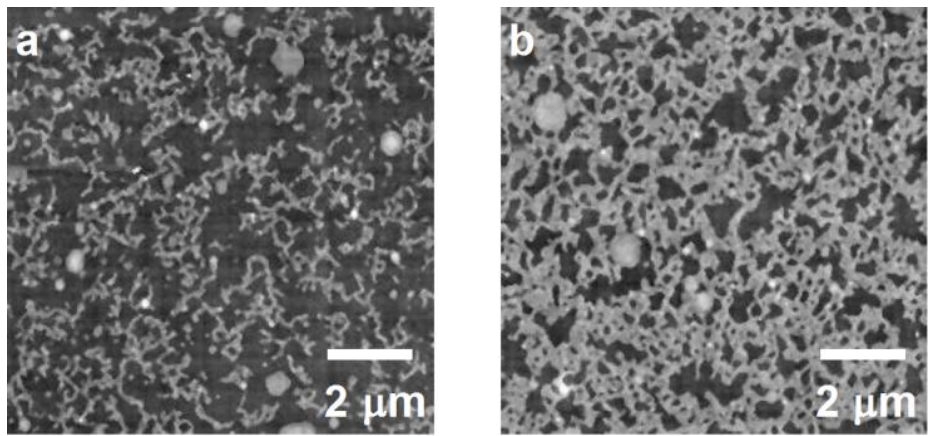

Figure 3. AFM images of PMPrS-g-PDEF 0.22 monolayers transferred at surface pressures of (a) $5 \mathrm{mN} / \mathrm{m}$ and (b) $20 \mathrm{mN} / \mathrm{m}$.

After AFM observation, we tried to build up LB films of PMPrS-g-PDEF. The first layer could be transferred onto a quartz substrate by the up-stroke with the transfer ratio ca. 1.0, but it was peeled off by the down-stroke in the next step. PDEF homopolymer has no ability to form multilayers, and consequently PMPrS- $g$-PDEF monolayers, whose interfacial behavior is strongly affected by PDEF graft chains, is also hard to be built up for LB films.

\subsection{Conformation of PMPrS in PMPrS-g-MAH spin-Coated Films}

The conformation of PMPrS main chains of PMPrS-g-MAH in the solid state was examined as in the case of PMPrS-g-PDEF. As shown in Figure 4, UV absorption spectra of PMPrS- $g$-MAH spin-coated films reveal that the fraction of the quasi-all-trans conformation indicated by the absorption peak at $325 \mathrm{~nm}$ decreases with an increase of grafting yield. When the ratio of the absorption intensity at $305 \mathrm{~nm}$ 
$\left(\mathrm{Abs}_{305}\right)$ to that at $325 \mathrm{~nm}\left(\mathrm{Abs}_{325}\right)$ is plotted against grafting yield, it decreases with increasing grafting yield as shown in Figure 5. This fact means that crystallization of PMPrS chains is hindered by grafted MAH units and the fraction of the quasi-all-trans conformation of PMPrS main chains decreases. In the case of PMPrS- $g$-PDEF, each of PMPrS and PDEF chains forms separate domains and the ratio of random and quasi-all-trans conformations is independent of the grafting yield as described in the former section. However, the graft chains of PMPrS-g-MAH are too short to form such separate domains. Thus, grafting yield directly affects the conformation of PMPrS main chains and the ratio of the absorption intensity is dependent on the grafting yield.

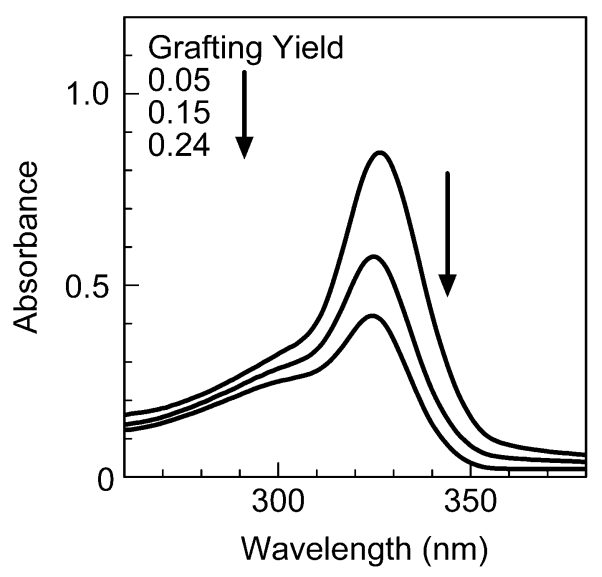

Figure 4. UV absorption spectra of spin-coated films of PMPrS- $g$-MAH. 


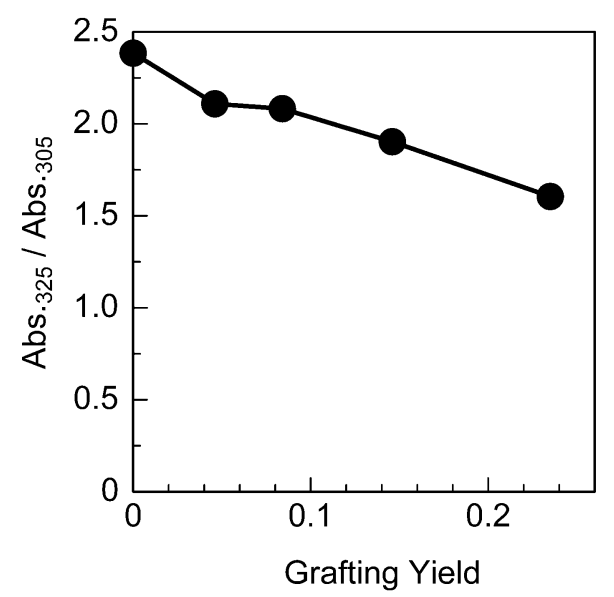

Figure 5. Relation between the ratio of the absorption intensity (Abs.325 / Abs.305) and grafting yield.

\subsection{Monolayers of PMPrS-g-MAH}

$\pi$-A isotherms of PMPrS-g-MAH monolayers are shown in Figure 6. Similarly to PMPrS-g-PDEF, PMPrS- $g$-MAH spreads more readily than PMPrS homopolymer does. However, the occupied area of PMPrS-g-MAH monolayers is smaller than that of PMPrS-g-PDEF monolayers with comparable grafting yield. We consider that this difference is attributed to the dissimilar structure of these two monolayers at the air/water interface due to the disparity in the length and density of the grafted chains. While the long PDEF chains of PMPrS- $g$-PDEF extensively spread on the water surface, MAH graft units are short and cannot spread so extensively as PDEF chains can. Besides, ungrafted parts of PMPrS chains between MAH-grafted parts are detached from the water surface. As a result, the occupied area of PMPrS-g-MAH monolayers is smaller than that of PMPrS- $g$-PDEF monolayers. At high grafting yields, however, PMPrS-g-MAH monolayers spread extensively enough. This fact suggests that the 
PMPrS chains are firmly confined on the water surface by densely grafted MAH units.

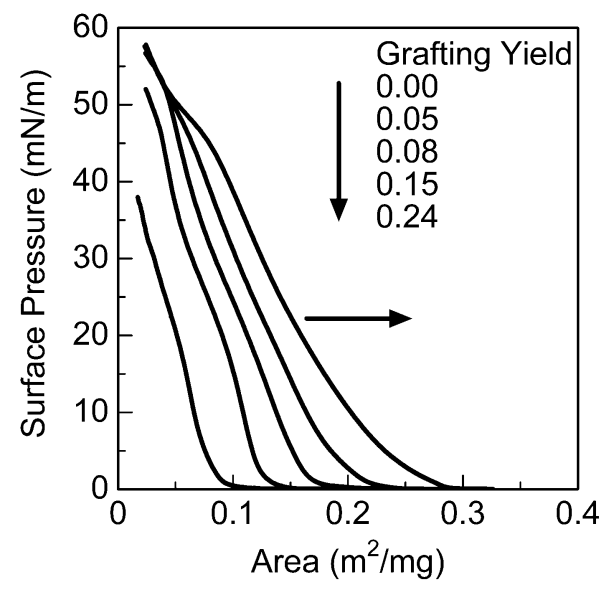

Figure 6. $\pi$-A isotherms of PMPrS- $g$-MAH monolayers at the air/water interface.

It is important to know the collapse pressure of monolayers at the air/water interface when the monolayers are transferred to solid substrates, but PMPrS- $g$-MAH monolayers do not show definite collapse pressure as shown in Figure 6. Accordingly, we judged the collapse pressure by examining the hysteresis of $\pi$-A isotherms of a PMPrS- $g$-MAH monolayer in a compression-expansion process. The $\pi$-A isotherm of a PMPrS- $g-\mathrm{MAH}_{0.24}$ monolayer on the second compression indicates the same profile as that on the first compression up to $35 \mathrm{mN} / \mathrm{m}$. When the monolayer is compressed up to $50 \mathrm{mN} / \mathrm{m}$, however, the $\pi$-A isotherm shows no reproducibility, which indicates collapse of the monolayer. Therefore, we assume that the bending points around the surface pressure of $50 \mathrm{mN} / \mathrm{m}$ on the $\pi$-A isotherms in Figure 6 indicate the collapse pressure of the monolayers.

A typical AFM image of a PMPrS- $g$ - $\mathrm{MAH}_{0.24}$ monolayer transferred onto a quartz 
substrate below the collapse pressure is displayed in Figure 7. PMPrS- $g$-MAH forms a uniform monolayer in contrast to PMPrS- $g$-PDEF as shown in Figure 3 because hydrophobic PMPrS chains are confined in the two-dimensional interface by MAH graft units acting like anchors and fully spread on the water surface.

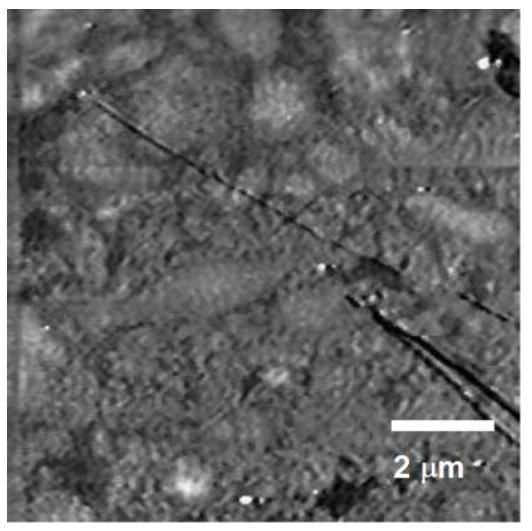

Figure 7. AFM image of a PMPrS-g- $\mathrm{MAH}_{0.24}$ monolayer transferred at a surface pressure of $5 \mathrm{mN} / \mathrm{m}$.

In situ UV absorption spectra of PMPrS- $g$-MAH monolayers at the air/water interface are depicted in Figure 8. Both for PMPrS- $g-\mathrm{MAH}_{0.15}$ and PMPrS-g-MAH $\mathrm{MA.24}$ monolayers, the absorption intensity entirely rises with increasing surface pressure because the surface density of the monolayers is heightened by the compression. However, comparing the spectra in detail, it is found that the absorption around $305 \mathrm{~nm}$ of PMPrS-g-MAH $\mathrm{M}_{0.24}$ monolayers is larger than that of PMPrS- $g$-MAH $\mathrm{MA}_{0.15}$ monolayers especially at lower surface pressures, which means the larger fraction of random conformation of PMPrS main chains in PMPrS- $g-\mathrm{MAH}_{0.24}$ monolayers. In the case of PMPrS- $g$-MAH with lower grafting yield, ungrafted PMPrS chains are detached from the water surface and crystallize by aggregating together. On the other hand, for 
PMPrS-g-MAH with higher grafting yield, PMPrS chains are more firmly confined on the water surface, and therefore hardly aggregate for crystallization. Nevertheless, the fraction of the quasi-all-trans conformation of PMPrS- $g-\mathrm{MAH}_{0.24}$, which corresponds to the relative absorption intensity around $325 \mathrm{~nm}$, rises with increasing surface pressure in Figure 8. One reason for this is that the two-dimensional crystallization occurs on compression of the monolayer. PMPrS chains are prone to align perpendicular to the compression direction. As a result, the PMPrS chains are forced to take the quasi-all-trans conformation.
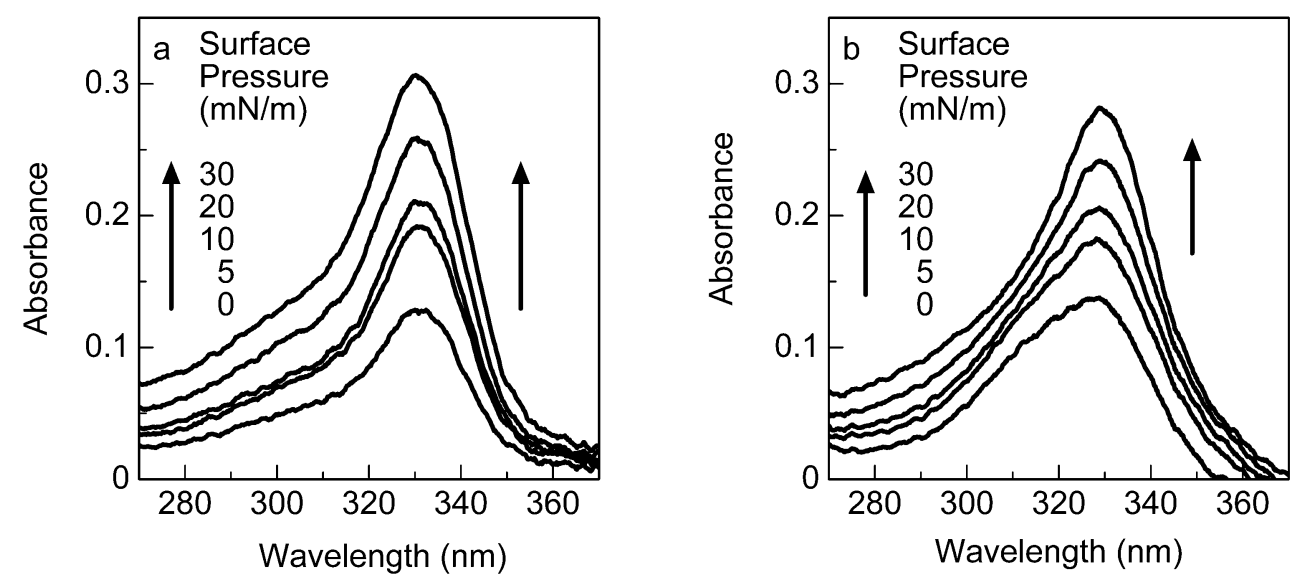

Figure 8. In-situ UV absorption spectra of (a) PMPrS-g-MAH $\mathrm{H}_{0.15}$ and (b) PMPrS-g-MAH $\mathrm{M}_{0.24}$ monolayers at the air/water interface.

Here, the thickness of the PMPrS-g-MAH monolayers is estimated to verify the monolayer structure. The thickness, $D$, is calculated by the following equation: $D=1$ / $\left(d_{\mathrm{Si}} w_{\mathrm{Si}}+d_{\mathrm{MAH}} w_{\mathrm{MAH}}\right) A$. In this equation, $d_{\mathrm{Si}}$ and $d_{\mathrm{MAH}}$ are the mass densities of $\operatorname{PMPrS}\left(1.14 \mathrm{~g} / \mathrm{cm}^{3}\right)$ and MAH $\left(1.48 \mathrm{~g} / \mathrm{cm}^{3}\right)$ in the bulk state [26,27], respectively, $w_{\mathrm{Si}}$ 
and $w_{\text {MAH }}$ are the weight fractions of PMPrS graft chains and MAH graft units, respectively, and $A$ is the occupied surface area of a PMPrS- $g$-MAH monolayer. The result of the calculation is listed in Table 2. The thickness decreases with an increase of grafting yield. This fact strongly suggests that the densely grafted PMPrS-g-MAH can spread more extensively and the PMPrS chains are firmly confined on the water surface by grafted MAH units.

Table 2. Thickness of the PMPrS- $g$-MAH monolayers.

\begin{tabular}{cccc}
\hline Surface pressure & PMPrS- $g$-MAH & & PMPrS- $g$-MAH \\
$(\mathrm{mN} / \mathrm{m})$ & $D(\mathrm{~nm})$ & $D(\mathrm{~nm})$ & PMPrS- $-\mathrm{MAH}_{0.24}$ \\
\cline { 2 - 4 } & 5.6 & 4.5 & $D(\mathrm{~nm})$ \\
\hline 5 & 7.6 & 6.3 & 3.6 \\
20 & 12 & 9.4 & 5.2 \\
35 & 14 & 11 & 8.5 \\
\hline 0 & & & \\
\hline
\end{tabular}

\subsection{LB films of PMPrS-g-MAH}

While PMPrS- $g$-PDEF monolayers did not form multilayer films, PMPrS- $g$-MAH ${ }_{0.24}$ monolayers were successfully deposited layer-by-layer on a solid substrate by the Y-type deposition. The successful layer-by-layer deposition was confirmed by UV absorption spectra of built-up LB films, as shown in Figure 9. The entire absorption intensities proportionally rise with increasing number of layers. The inset of Figure 9 shows the nearly linear increase of absorbance at $325 \mathrm{~nm}$ against the number of layers. 


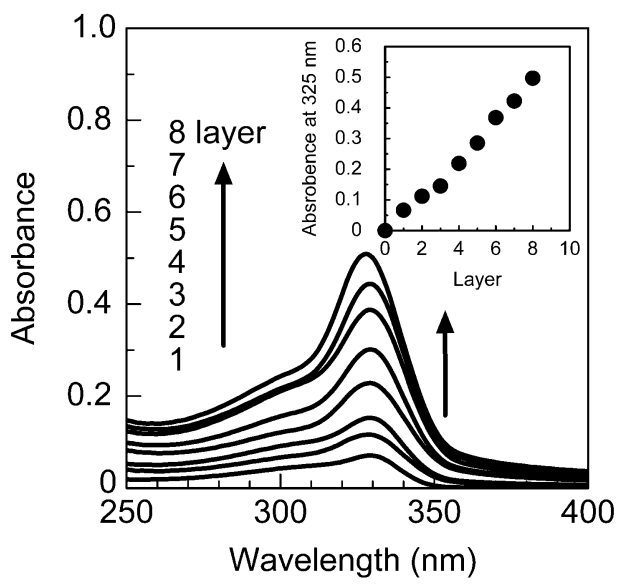

Figure 9. UV absorption spectra of PMPrS-g-MAH $\mathrm{H}_{0.24} \mathrm{LB}$ films. The inset is a plot of absorbance at $325 \mathrm{~nm}$ as a function of the number of layers.

The relationship between the transfer ratio and the number of layers of a PMPrS- $g-\mathrm{MAH}_{0.24} \mathrm{LB}$ film was investigated. Up to 10 layers, the transfer ratios were $1.0 \pm 0.2$ for up-strokes and $0.7 \pm 0.1$ for down-strokes, which implies that the deposition by hydrophilic interaction of MAH is more stable than that by hydrophobic interaction by PMPrS. It can be said from the estimated thickness of Table 2 that the PMPrS- $g$-MAH LB film is also a nano-scale structure.

\section{Conclusion}

In this study, we clearly demonstrated that the grafting manner of radiation-modified amphiphilic polysilanes has a significant effect on the morphology of their monolayers. At the air/water interface, both PMPrS- $g$-PDEF and PMPrS- $g$-MAH form monolayers, but the monolayers of PMPrS- $g$-PDEF and PMPrS- $g$-MAH have quite dissimilar morphology: PMPrS- $g$-PDEF forms inhomogeneous monolayers with string-like 
microstructures, while the PMPrS-g-MAH monolayers are more uniform. It seems that higher grafting yield indicating more hydrophilic groups in the polymer leads to more uniform monolayers. However the present study reveals that the key factor to give uniform monolayers is the density of graft chains on polymers, not the grafting yield and the length of graft chains. In most cases, graft and block copolymers composed of hydrophobic and hydrophilic chains form inhomogeneous monolayers or monolayers with regularly-structured surfaces [28-36]. Even for copolymers composed of hydrophilic chains alone, those monolayers often exhibit inhomogeneous feature due to phase separation [37]. There have been few reports on the uniform monolayers formed with amphiphilically-modified copolymers. However, the relationship between monolayer uniformity and the structure of graft copolymers clarified here offers a new principle for preparing monolayer-forming polymers.

Our findings also provide useful information on the fabrication of high quality LB films of amphiphilic polysilanes. The interfacial property of amphiphilically grafted polysilanes inherits that of grafted chains, as seen in the layer-by-layer deposition of the LB films. Therefore, appropriate selection of grafted monomers proves to be an important factor to utilize radiation-modification for the fabrication of polysilane nano-ordered materials.

\section{Acknowledgments}

We thank Prof. S. Ito and Prof. H. Aoki (Department of Polymer Chemistry, Kyoto University) for AFM measurements, Prof. H. Yamana and Prof. T. Fujii (Research Reactor Institute, Kyoto University) for in-situ UV spectroscopy. 


\section{References}

[1] R. J. West, J. Organomet. Chem. 300 (1986) 327.

[2] R. D. Miller, J. Michl, Chem. Rev. 89 (1989) 1359.

[3] M. Fujino, Chem. Phys. Lett. 136 (1987) 451.

[4] R. G. Kepler, J. M. Zeigler, L. A. Harrah, S. R. Kurtz, Phys. Rev. B35 (1987) 2818.

[5] M. Stolka, H. J. Yuh, K. McGrane, D. M. Pai, J. Polym. Sci., Polym. Chem. 25 (1987) 823.

[6] M. A. Abkowitz, F. E. Knier, H. J. Yuh, R.J. Weagley, M. Stolka, Solid State Commun. 62 (1987) 547.

[7] C. H. Yuan, S. Hoshino, S. Toyoda, H. Suzuki, M. Fujiki, N. Matsumoto, Appl. Phys. Lett. 71 (1997) 3326.

[8] H. Suzuki, S. Hoshino, K. Furukawa, K. Ebata, C. H. Yuan, I. Bleyl, Polym. Adv. Technol. 11 (2000) 460.

[9] L. M. Samuel, P. N. Sanda, R. D. Miller, Chem. Phys. Lett. 159 (1989) 227.

[10] K. Yokoyama, M. Yokoyama, Chem. Lett. 1989, 1005.

[11] S. Mitter-Neher, D. Neher, G. I. Stegeman, F. W. Embs, G. Wegner, Chem. Phys. 161 (1992) 289.

[12] F. Kajar, J. Messier, C. Rosilio, J. Appl. Phys. 60 (1986) 3040.

[13] R. West, In Inorganic Polymers; J. E. Mark, H. R. Allcock, R. West, Prentice Hall: Englewood Cliffs, NJ, 1992; Chapter5, p 186.

[14] W. Chunwachirasiri, I. Kanaglekar, M. J. Winokur, J. C. Koe, R. West, Macromolecules 34 (2001) 6719.

[15] S. Nagano, T. Seki, K. Ichimura, Langmuir 17 (2001) 2199.

[16] S. Nagano, T. Seki, J. Am. Chem. Soc. 124 (2002) 2074. 
[17] M. Fujiki, J. R. Koe, K. Terao, T. Sato, A. Teramoto, J. Watanabe, Polym. J. 35 (2003) 297.

[18] H. Tanaka, N. Sato, T. Matsuyama, Langmuir 21 (2005) 7696.

[19] H. Tanaka, N. Sato, T. Matsuyama, R. Inoue, T. Kanaya, Polym. J. 39 (2007) 874.

[20] F. W. Embs, G. Wegner, D. Neher, P. Albouy, R. D. Miller, C. G. Willson, W. Schrepp, Macromolecules 24 (1991) 5068.

[21] H. Yoshida, R. Kani, S. Hayase, K. Horie, J. Phys. Chem. 97 (1993) 5370.

[22] R. Kani, Y. Nakano, Y. Majima, S. Hayase, C. H. Yuan, R. West, Macromolecule 27 (1994) 1911.

[23] R. Kani, H. Yoshida, Y. Nakano, S. Marui, Y Mori, Y. Kawata, S. Hayase, Langmuir 9 (1993) 3045.

[24] T. Seki, N. Tanigaki, K. Yase, A. Kaito, T. Tamaki, K. Ueno, Y. Tanaka, Macromolecules 28 (1995) 5609.

[25] H. Tanaka, I. Iwasaki, Y. Kunai, N. Sato, T. Matsuyama, Radiat. Phys. Chem. 80 (2011) 884 .

[26] J. Leonard, In Polymer Handbook 4th Edition; J. Brandrup, E. H. Immergut, E. A. Grulke, (Eds.), Wiley, New York, 1999.

[27] B. Jambe, A. Jonas, J. Devaux, J. Polym. Sci. B. Polym. Phys. 35 (1997) 1533.

[28] N. Gabriel, L. C. Njikang, M. Gauthier, Langmuir 24 (2008) 12919.

[29] Solvents and Self-Organization of Polymers; S. E. Webber, P. Munk, Z. Tuzar, (Eds.), Kluwer, The Netherlands, 1996.

[28] N. Gabriel, L. C. Njikang, M. Gauthier, Langmuir 24 (2008) 12919.

[29] B. H. Cao, M. W. Kim, D. G. Peiffed, Langmuir 11 (1995) 1645.

[30] Solvents and Self-Organization of Polymers; S. E. Webber, P. Munk, Z. Tuzar, 
(Eds.), Kluwer, The Netherlands, 1996.

[31] J. Zhu, R. B. Lennox, A. Eisenberg, Langmuir 7 (1991) 1579.

[32] S. Li, S. Hanley, I. Khan, S. K. Varshney, A. Eisenberg, R. B. Lennox, Langmuir 9 (1993) 2243.

[33] Y. Seo, J. H. Im, J. S. Lee, J. H. Kim, Macromolecules 34 (2001) 4842.

[34] Y. Seo, K. Paeng, S. Park, Macromolecules 34 (2001) 8735.

[35] C. A. Devereaux, S. M. Baker, Macromolecules 35 (2002) 1921.

[36] Y. Seo, A. R. Esker, D. Sohn, H. J. Kim, S. Park, H. Yu, Langmuir 19 (2003) 3313.

[37] J. Kumaki, T. Hashimoto, J. Am. Chem. Soc. 120 (1998) 423. 


\section{Figure Captions}

Figure 1. Apparatus for in-situ UV absorption spectroscopy of a monolayer at the air/water interface.

Figure 2. $\pi$-A isotherms of PMPrS- $g$-PDEF monolayers at the air/water interface.

Figure 3. AFM images of PMPrS-g-PDEF ${ }_{0.22}$ monolayers transferred at surface pressures of (a) $5 \mathrm{mN} / \mathrm{m}$ and (b) $20 \mathrm{mN} / \mathrm{m}$.

Figure 4. UV absorption spectra of spin-coated films of PMPrS-g-MAH.

Figure 5. Relation between the ratio of the absorption intensity (Abs.325/Abs.305) and grafting yield.

Figure 6. $\pi$-A isotherms of PMPrS- $g$-MAH monolayers at the air/water interface.

Figure 7. AFM image of a PMPrS-g- $\mathrm{MAH}_{0.24}$ monolayer transferred at a surface pressure of $5 \mathrm{mN} / \mathrm{m}$.

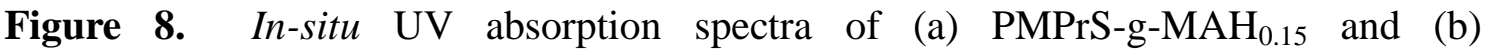
PMPrS-g-MAH $\mathrm{M}_{0.24}$ monolayers at the air/water interface.

Figure 9. UV absorption spectra of PMPrS-g-MAH $\mathrm{MA}_{0.24} \mathrm{LB}$ films. The inset is a plot of absorbance at $325 \mathrm{~nm}$ as a function of the number of layers. 